\title{
THE BROADENING OF THE OPTICAL PARAMETRIC AMPLIFICATION BANDWIDTH CAUSED BY IDLER WAVE ABSORPTION
}

\author{
G. Valiulis and A. Varanavičius \\ Laser Research Center, Vilnius University, Sauletekio 10, 10223 Vilnius, Lithuania \\ Email: gintaras.valiulis@ff.vu.lt
}

Received 21 May 2021; revised 30 August 2021; accepted 1 September 2021

\begin{abstract}
The amplification bandwidth of an optical parametric amplifier (OPA) defines the duration of the signal and idler pulse. Usually the OPA bandwidth is described by material dispersion and interaction geometry. The absorption of interacting waves is detrimental to the amplification process. However, even in the case of a large linear absorption of the idler wave it is still possible to get an effective amplification and a positive effect to the amplifier bandwidth. In the current work, we investigate the influence of idler wave linear losses on the OPA bandwidth.
\end{abstract}

Keywords: optical parametric amplification, absorption

PACS: $42.65 . \mathrm{Ky}, 42.65 . \mathrm{Yj}, 42.25 . \mathrm{Bs}, 42.79 . \mathrm{Nv}$

\section{Introduction}

The optical parametric amplification process is an effective method to generate tunable light radiation and can provide large amplification bandwidths needed for the formation of ultrashort (a few femtosecond) light pulses [1]. The waves amplified by pump wave (higher carrier frequency wave) in an optical parametric amplifier (OPA) are called a signal and an idler, and both of them can be useful for applications. Usually, it is a common opinion that the absorption of signal and idler waves is a detrimental phenomenon, limiting the efficiency of the OPA. A couple of decades ago, Lowenthal studied the influence of idler wave absorption on the OPA process in a continuous wave regime. This study showed that some idler absorption might even improve the signal output power [2]. The pulsed OPA regime was studied by Lyons et al. for a singly resonant OPA through numerical simulations and it was found that back conversion could be significantly affected by idler absorption [3]. In Ref. [4], it was demonstrated numerically that OPA with a large absorption of the idler beam might perform better than without idler absorption in terms of both signal conversion efficiency and signal beam quality due to the reason that idler absorption can suppress back conversion. The chirped pulse amplification was investigated also experimentally in a $\mathrm{Sm}^{3+}$-doped yttrium calcium oxyborate crystal [5]. In this work, we present an analysis of how idler absorption affects the amplification bandwidth of OPA.

\section{The bandwidth of OPA}

In order to show the influence of absorption on the bandwith of optical parametric amplification (OPA), we will start with the equations describing the OPA process

$$
\begin{gathered}
\frac{\partial A_{1}}{\partial z}+v_{13} \frac{\partial A_{1}}{\partial t}-\frac{\mathrm{i}}{2} g_{1} \frac{\partial^{2} A_{1}}{\partial t^{2}}=-\mathrm{i} \sigma_{1} A_{2}^{*} A_{3}, \\
\frac{\partial A_{2}}{\partial z}+v_{23} \frac{\partial A_{2}}{\partial t}-\frac{\mathrm{i}}{2} g_{2} \frac{\partial^{2} A_{2}}{\partial t^{2}}=-\mathrm{i} \sigma_{2} A_{1}^{*} A_{3}, \\
\frac{\partial A_{3}}{\partial z}-\frac{\mathrm{i}}{2} g_{3} \frac{\partial^{2} A_{3}}{\partial t^{2}}=-\mathrm{i} \sigma_{1} A_{1} A_{2},
\end{gathered}
$$

where $A_{j}(t, z)$ is the wave complex amplitude, $t$ is the retarded time (corresponding to the coordi- 
nate frame moving with the pump wave), and $z$ is the longitudinal coordinate. $j=1,2$ and 3 stand for signal, idler and pump waves, respectively. $v_{j 3}=u_{j}^{-1}-u_{3}^{-1}$ are the coefficients of group velocity mismatch $(\mathrm{GVM}), u_{j}$ is the group velocity of wave $j$ calculated at the carrier frequency $\omega_{j 0}$, and for the carrier frequencies we have the constraint $\omega_{10}+\omega_{20}=\omega_{30}$, typical of the three-wave interaction process. Parameters $g_{j}=\mathrm{d}^{2} k /\left.\mathrm{d} \omega^{2}\right|_{\omega_{\mathrm{j}}}$ are the coefficients of group velocity dispersion (GVD), $k_{j}$ is the absolute value of the wave $j$ axial wave-vector, and $\sigma_{j}$ is the nonlinear coupling coefficient. Note that the material dispersion is taken into account up to the second order.

In the case of a plane, monochromatic and undepleted pump wave $\left(\left|A_{1}\right|,\left|A_{2}\right| \ll\left|A_{3}\right|, A_{3}=\right.$ const. $=a_{3}$ ) instead of Eq. (1) we have a system of two linear equations:

$$
\begin{aligned}
& \frac{\partial A_{1}}{\partial z}+v_{13} \frac{\partial A_{1}}{\partial t}-\frac{\mathrm{i}}{2} g_{1} \frac{\partial^{2} A_{1}}{\partial t^{2}}=-\mathrm{i} \sigma_{1} A_{2}^{*} A_{3}, \\
& \frac{\partial A_{2}}{\partial z}+v_{23} \frac{\partial A_{2}}{\partial t}-\frac{\mathrm{i}}{2} g_{2} \frac{\partial^{2} A_{2}}{\partial t^{2}}=-\mathrm{i} \sigma_{2} A_{1}^{*} A_{3},
\end{aligned}
$$

The solution of the set of equations (2) can be found in the spectral domain using the Fourier transform

$$
A_{j}(t, z)=\frac{1}{2 \pi} \int_{-\infty}^{\infty} S_{j}(\omega, z) \mathrm{e}^{\mathrm{i} \omega t} \mathrm{~d} \omega .
$$

By substituting this expression for $A$ in Eqs. (2) we obtain the set of ordinary differential equations in the spectral domain. The exact analytic solution of these equations can be found. In the case of a large gain, when $\Gamma_{0} z \gg 1\left(\Gamma_{0}=\sqrt{\sigma_{1} \sigma_{2} a_{30}}\right)$ and when $A_{20}(t)=A_{2}(t, z=0)=0, S_{20}(\omega)=S_{2}(\omega, z=0)=0$, the approximate solution (assuming $\Gamma_{0} z \gg 1$ ) is

$$
\begin{aligned}
S_{1}(\omega, z) \approx & \frac{1}{2} S_{10}(\omega) \exp (\Gamma(\omega) z) \exp (-\mathrm{i} D(\omega) z), \\
& S_{2}^{*}(-\omega, z) \approx \mathrm{i} \sqrt{\frac{\sigma_{2}}{\sigma_{1}}} S_{1}(\omega, z)
\end{aligned}
$$

with the introduced coefficients

$$
\begin{aligned}
& \Gamma(\omega)=\sqrt{\Gamma_{0}^{2}-K(\omega)^{2}}, \\
& K(\omega)=\frac{1}{2}\left(v_{12} \omega+\frac{g_{1}+g_{2}}{2} \omega^{2}\right),
\end{aligned}
$$

$$
D(\omega)=\frac{v_{13}+v_{23}}{2} \omega+\frac{g_{1}-g_{2}}{2} \omega^{2},
$$

where $\Gamma_{0}=\sqrt{\sigma_{1} \sigma_{2}} a_{30}$ is the parametric gain increment for the case of plane and monochromatic waves. From the analysis of Eqs. (4-7) the following conclusions can be done.

First, as can be seen from Eqs. (4), the signal and idler waves are conjugated (the second equation of Eqs. (4) in the temporal domain corresponds to $A_{2}(t, z) \approx \mathrm{i} \sqrt{\frac{\sigma_{2}}{\sigma_{1}}} A_{1}^{*}(t, z)$, thus we have the phase conjugation for signal and idler waves: $\varphi_{2}(t, z)=$ $-\varphi_{1}(t, \mathrm{z})$ (also known as chirp reversal phenomena).

Second, the parameter $D(\omega)$, i.e. the so-called nonlinear dispersion, describes the evolution of the spectral phase during propagation, whereas the parameter $\Gamma(\omega)$ defines the gain of spectral components. The gain bandwidth of the parametric amplifier becomes infinite if the gain for all the spectral components is uniform: $\Gamma(\omega)=$ const. This condition requires $K(\omega)=0$. Such condition could be satisfied in OPA with angularly dispersed waves (X pulses, for example) [6]. In collinear OPA, without wave front tilting and a large beam diameter, the condition $K(\omega)=0$ becomes impossible due to the material dispersion and a narrow angular spectrum. It can be approximately fulfilled just in the vicinity of the carrier frequency. However, if the width of the gain $\Gamma(\omega)$ is much larger than the bandwidth of the amplified pulse, all spectral components of the pulse have a nearly uniform amplification. By using Eqs. (5) and (6) under some assumptions, and considering just the first dispersion approximation $\left(K_{0}(\omega)=1 / 2\left(v_{12} \omega\right)\right)$, it is possible to derive the well-known expression for the OPA amplification bandwidth $\Delta \omega_{\text {opa }}$ :

$$
\Delta \omega_{\mathrm{opa}}=4 \sqrt{\frac{\ln 2 \Gamma_{0}}{v_{12}^{2} z}} .
$$

As can be seen from Eq. (8), the OPA bandwidth within the first dispersion approximation is defined by the group mismatch $v_{12}$ between signal and idler pulses, which becomes negligible near the type I degenerate phase matching $\omega_{10} \approx \omega_{20}$, and, consequently, the second-order dispersion becomes the factor that limits the bandwidth of OPA. However, far from a degenerate interaction the group 
velocity mismatch between signal and idler waves is the major factor limiting the amplification bandwidth.

Note that the pulse amplified in OPA possesses some extra dispersion that does not coincide with the material dispersion, or in other words, is modified by nonlinearity. That so-called nonlinear dispersion is taken into account via the parameter $D(\omega)$. As can be seen from Eq. (7), due to that circumstance, both signal and idler waves propagate with the same group velocity (defined by the averaged group mismatch parameter $\left.\left(v_{13}+v_{23}\right) / 2\right)$, having suppressed GVD (if both GVD parameters $g_{1}$ and $g_{2}$ are of the same sign). Near type-I degenerate phase matching $g_{1} \approx g_{2}$, thus that suppressed GVD both for the signal and the idler becomes nearly zero $\left(\frac{g_{1}-g_{2}}{2} \rightarrow 0\right)$. However, for extremely short pulses the averaged third-order dispersion is common for both signal and idler waves and should be taken into account for OPA.

\section{The influence of linear absorption on the OPA bandwidth}

Let us discuss the influence of linear absorption on the parametric amplification process. To take dissipation into account in the spectral domain we simply need to modify the parameters $K(\omega)$ and $D(\omega)$ by adding imaginary terms responsible for the absorption of signal and idler waves. In the case of frequency-dependent absorption this corresponds to the inclusion of extra terms $\int_{0}^{\infty} \bar{\alpha}_{j}(\tau) A_{j}(t-\tau) \mathrm{d} \tau$ (where $\left.\bar{\alpha}_{j}(t)=\frac{1}{2 \pi} \int_{-\infty}^{+\infty} \alpha_{j}(\omega) \mathrm{e}^{-\mathrm{i} \omega t} \mathrm{~d} \omega\right)$ in the left side of equations (2), or simply just the $\alpha_{j} A_{j}$ term for the case of nondispersive dissipation $\left(\alpha_{j}(\omega)=\alpha_{j}\right)$. Thus, in the dissipative media the parameters $K(\omega)$ and $D(\omega)$ become complex:

$$
\begin{aligned}
& K(\omega)=K_{0}(\omega)+\frac{\mathrm{i}}{2}\left[\alpha_{2}(\omega)-\alpha_{1}(\omega)\right], \\
& D(\omega)=D_{0}(\omega)-\frac{\mathrm{i}}{2}\left[\alpha_{2}(\omega)+\alpha_{1}(\omega)\right] .
\end{aligned}
$$

Here $K_{0}(\omega)$ and $D_{0}(\omega)$ denote the same coefficients as in the non-dissipative medium:

$$
K_{0}(\omega)=\frac{1}{2}\left(v_{12} \omega+\frac{g_{1}+g_{2}}{2} \omega^{2}\right),
$$

$$
D_{0}(\omega)=\frac{v_{13}+v_{23}}{2} \omega+\frac{g_{1}-g_{2}}{2} \omega^{2} .
$$

So linear absorption is taken into account via the coefficients $\alpha_{1}(\omega)$ and $\alpha_{2}(\omega)$ calculated at signal and idler frequencies: $\alpha_{1}(\omega)=\alpha\left(\omega_{10}+\omega\right)$ and $\alpha_{2}(\omega)=\alpha\left(\omega_{20}-\omega\right)$, where $\alpha(\omega)$ is the frequencydependent linear absorption coefficient of the medium.

In the non-dissipative medium, the parameter $K(\omega)=K_{0}(\omega)$ defines the gain dependence on the frequency $\omega$, i.e. the amplification bandwidth, and parameter $D(\omega)=D_{0}(\omega)$ defines the spectral phase dependence on the frequency $\omega$, i.e. the nonlinear dispersion emerging in OPA. The linear absorption makes these parameters complex quantities, thus the parameter $K(\omega)$ gives the imaginary term to the gain parameter $\Gamma(\omega)$ and in such a way affects the spectral phase. In a similar way, the imaginary part of $D(\omega)$ affects the gain. However, if the absorption coefficients for signal and idler waves are nearly equal, $\alpha_{1} \approx \alpha_{2} \approx \alpha$, just the imaginary part of $D(\omega)$ becomes significant and gives the reduction of the gain both to the signal and idler waves (since $\Gamma(\omega)$ remains unmodified, $D(\omega) \sim-\mathrm{i} \alpha(\omega)$ gives an extra term to $G(\omega)$ ):

$$
\begin{aligned}
& S_{1}(\omega, z) \approx \frac{1}{2} S_{10}(\omega) \\
& \exp \left(-\mathrm{i} D_{0}(\omega) z\right) \exp \{G(\omega) z\}, \\
& G(\omega)=\sqrt{\Gamma_{0}^{2}-K_{0}(\omega)^{2}}-\alpha(\omega) .
\end{aligned}
$$

As one can see, the gain increment $G(\omega)$ contains the term $-\alpha(\omega)$ responsible for the gain reduction both for signal and idler waves.

Let us focus now on the case of amplification far from degeneracy when the idler waves slip out of a transmission window while the losses for the pump and signal are negligible. For this case, we assume that $\alpha_{2} \gg \alpha_{1} \approx 0$. As can be seen from the analysis of the solution in the case of non-symmetric losses, the OPA possesses some interesting features. In order to demonstrate these features, we recast Eq. (4) by using Eq. (5) and substituting Eqs (9-12):

$$
\begin{aligned}
& S_{1}(\omega, z) \approx \frac{1}{2} S_{10}(\omega) \\
& \exp \left(-\mathrm{i} D_{0}(\omega) z\right) \exp \{G(\omega) z\},
\end{aligned}
$$




$$
G(\omega)=\sqrt{\Gamma_{0}{ }^{2}-\left[K_{0}(\omega)+\frac{\mathrm{i}}{2} \alpha_{2}(\omega)\right]^{2}}-\frac{1}{2} \alpha_{2}(\omega) .
$$

Equation (15) remains unchanged (as Eq. (13)), but $G(\omega)$ incurs significant modifications. Several conclusions can be drawn.

Since $K_{0}(0)=0$, the gain coefficient $G(\omega)$ remains positive regardless of the amount of idler absorption.

In the case of $K_{0}(\omega) \ll \alpha_{2}(\omega)$ when the idler wave absorption is dominant over material dispersion, instead of Eq. (16) we have

$$
G(\omega) \approx \sqrt{\Gamma_{0}^{2}+\frac{1}{4} \alpha_{2}(\omega)^{2}}-\frac{1}{2} \alpha_{2}(\omega) .
$$

In the current situation, the material dispersion does not define anymore the gain spectral width (via coefficient $K_{0}(\omega)$ ) and the OPA bandwidth, respectively. Moreover, even in the case of a very large absorption $\left(\Gamma_{0} \ll \alpha_{2}\right)$, the OPA still performs the exponential amplification:

$$
S_{1}(\omega, z) \sim \exp \left(-i D_{0}(\omega) z\right) \exp \left\{\Gamma_{0} z \frac{\Gamma_{0}}{\alpha_{2}(\omega)}\right\} .
$$

However, as can be seen from Eq. (17), for a very large absorption the OPA gain coefficient drops from the maximum possible value $\Gamma_{0} z$ by the factor $\frac{\Gamma_{0}}{\alpha_{2}(\omega)}$. Despite that, the same amplification as in a lossless medium can also be achieved in the absorbing material, but in a longer propagation path.

In the case when idler wave absorption is large and nearly independent of frequency $\left(K_{0}(\omega) \ll \alpha_{2}(\omega)=\alpha_{2}=\right.$ const. $)$, the gain during parametric amplification becomes also nearly independent of the frequency $\omega$ (see Eqs $(16-18)$ ), i.e. the gain bandwidth becomes broader (or even unlimited).

Therefore, in the case of a large idler wave absorption a parametric amplifier sustains exponential gain with a broadened amplification bandwidth. This implies that despite large losses, pulse spectral components could be amplified to the same level as in the lossless medium.

\section{Numerical demonstration}

Since in the presence of absorption there is no possibility to derive an accurate equation (similar to Eq. (8)) for the bandwidth even taking into account the first dispersion approximation (when $\left.K_{0}(\omega)=1 / 2\left(v_{12} \omega\right)\right)$, the analytical results presented here have been reproduced by numerical simulations by means of a split-step [7] code solving the system of Eqs. (1) and taking into account the medium parameters. The crystal was divided into a number of slices. Each slice was passed twice: linear effects (diffraction, material dispersion and linear loses) were taken into account during the first pass, while the material nonlinearity during the second pass. The linear wave propagation was handled in the Fourier space, however the nonlinear part in the near field using the fourth-order Runge-Kutta method.

The group-velocity-mismatch parameter $v_{j 3}$ was taken into account via the group-velocity-mismatch length $L_{j 3}=\tau_{0} / v_{j 3}$ calculated for the $\tau_{0}=1 \mathrm{ps}$ pulse, and the group-velocity-dispersion parameter $g_{j}$ via the GVD length $L_{g_{j}}=\tau_{0}^{2} /\left(4 \ln 2 g_{j}\right)$, which defines the distance over which the duration of the Gaussian $\tau_{0}=1$ ps pulse increases by a factor of $\sqrt{2}$. The nonlinearity of the medium was taken into acount by introducing the nonlinear length $L_{n}=\left(\Gamma_{0}\right)^{-1}$, where $A_{0}$ is the pulse amplitude corresponding to the pulse intensity of $1 \mathrm{GW} / \mathrm{cm}^{2}$. The parameters of linear losses $\alpha_{j}$ were taken into account through the absorption length $L_{\alpha_{j}}=\left(2 \alpha_{j}\right)^{-1}$, defined as the distance over which the intensity drops by a factor of e, due to the absorption in a linear medium.

Numerical simulations were performed using an artificial crystal with made-up dispersion, absorption losses and nonlinearity. The GVD was switched off for the possibility to compare the numerical results with the analytic formula of Eq. (8) as a reference point for the lossless case. The group velocity mismatch was taken into account via group-velocity-mismatch lengths: $L_{13}=-50 \mathrm{~mm}$ for the signal and $L_{23}=50 \mathrm{~mm}$ for the idler. The idler wave absorption was varied from $\alpha_{2}=0 \mathrm{~mm}^{-1}\left(L_{\alpha_{2}}=\infty \mathrm{mm}\right)$ to $\alpha_{2}=50 \mathrm{~mm}^{-1}\left(L_{\alpha_{2}}=0.01 \mathrm{~mm}\right)$. For the sake of clarity we chose the pump wavelength 515 and $607 \mathrm{~nm}$ for the signal. For the pump intensity $50 \mathrm{GW} / \mathrm{cm}^{2}$, the nonlinear length is $L_{n}=0.40 \mathrm{~mm}$. We recast Eq. (8) by use of the above defined characteristic lengths. Thus, for the amplification bandwidth (in $\mathrm{cm}^{-1}$ ) we have

$$
\Delta v_{\text {opa }}\left[\mathrm{cm}^{-1}\right]=\frac{2}{0.03 \pi} \frac{L_{12}}{L_{n}} \sqrt{\frac{\ln 2}{z / L_{n}}} .
$$


For the lossless medium with the values of the parameters presented above we obtain $\Delta v_{\text {opa }}=$ $435 \mathrm{~cm}^{-1}$ from Eq. (19).

The results of numerical calculations representing the influence of idler wave absorption on the amplification bandwidth are shown in Fig. 11. When performing the simulation, the signal amplification ratio of $10^{4}$ was fixed and the length of the crystal was gradually increased from 2.6 to $41.5 \mathrm{~mm}$ in accordance with raising of the absorption parameter $\alpha_{2}$ from 0 to $50 \mathrm{~mm}^{-1}$. The initial duration of the injected signal pulse was $10 \mathrm{fs}$. The amplification bandwidth dependence on $\alpha_{2}$ is shown in Fig. 1 (a black line). As can be seen for $\alpha_{2}=0$, we obtain the value $\Delta v_{\text {opa }}=430 \mathrm{~cm}^{-1}$ as predicted by Eq. (19). The bandwidth is broadening when the $\alpha_{2}$ value is increasing, and becomes more than 3 times larger when $\alpha_{2}=50 \mathrm{~mm}^{-1}$. The amplified signal pulse remains transform-limited, with the duration supported by the amplification bandwidth (see Fig. 2, a black line).

The results of simulations show that the gain bandwidth broadening of the case with the 5 times larger first-order dispersion is presented in the inset of Fig. 1. For the pump intensity $70 \mathrm{GW} / \mathrm{cm}^{2}$, the nonlinear length is $L_{n}=0.34 \mathrm{~mm}$. Equation (19) gives the value $\Delta v_{\text {opa }}=90 \mathrm{~cm}^{-1}$ in this case. It should be noticed that the effect of bandwidth broadening

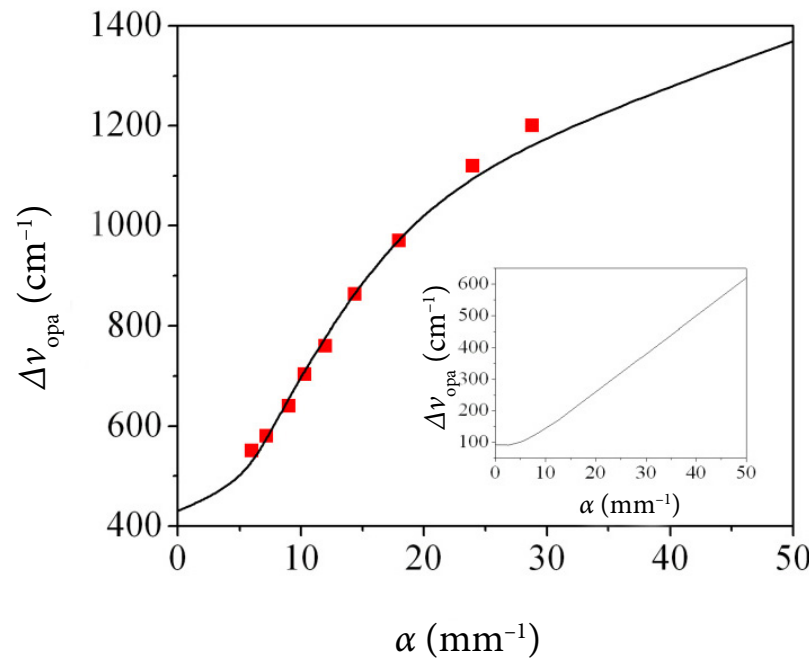

Fig. 1. Dependence of the amplification bandwidth on the absorption coefficient. Squares (red online) represent the results of simulation using multi-pass configuration. The inset: OPA bandwidth dependence on the absorption coefficient for the case with 5 times larger dispersion. See the text for details.

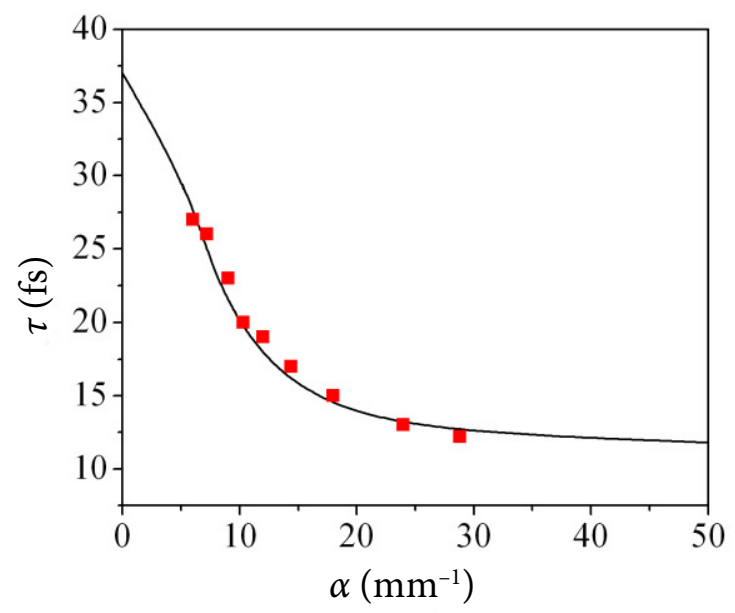

Fig. 2. Dependence of the signal pulse duration on the absorption coefficient. Squares (red online) represent the results of simulation using multi-pass configuration. See the text for details.

also takes place within the second of even higher dispersion approximation, and the dynamics depends on a larger set of parameters.

However, there is another way to simulate parametric amplification in the case of large idler losses by using multi-pass OPA in a short crystal, every time quenching the idler wave at the output. The effective absorption can be simulated by shortening the crystal and increasing the number of passes (in order to get the same amplification). For the parameters chosen above we introduce the effective absorption coefficient for the multipass system: $\alpha_{2}^{\text {eff }}=3,6 / L_{p}$, where $L_{p}$ is the crystal length in $\mathrm{mm}$. We performed calculations with nine different $L_{p}$ values $(0.6,0.5,0.4,0.35,0.3,0.25,0.2,0.15$ and $0.125 \mathrm{~mm}$ ), increasing the number of passes from 6 to 104 in order to obtain the same amplification. The results are presented by red squares in Figs. 1 and 2. As can be seen from the multi-pass system, the results perfectly render the case of the singlepass system.

We should note that the analysis presented in the current work is performed for the case of undepleted OPA. Our preliminary results show that the idler wave absorption also significantly affects the performance of OPA when the energy transfer is not negligible. The losses imposed on the idler allow us to suspend the back-conversion process leading to higher energy conversion from pump to signal efficiency. These findings and discussion of the practical implementation deserve a separate paper. 


\section{Conclusions}

The results of the theoretical analysis of OPA performance suggest that the strong absorption of idler wave during optical parametric amplification sustains the exponential growth of the signal and affects the amplification bandwidth. The analytical expression describing the evolution of parametric spectral bandwidth dependence on the losses for idler wave has been derived. The theoretical treatment has been verified by the numerical simulation of OPA performance. The obtained results show that in the case of a large enough absorption the amplification bandwidth can be enlarged by several times, in a longer amplification path. The same effect can be rendered by the use of the multi-pass system composed of a set of short optical crystals, by attenuation of the idler wave at the exit of each crystal.

\section{Acknowledgements}

This research was funded by the European Regional Development Fund according to the supported activity 'Research Projects Implemented by World-class Researcher Groups' under Measure No. 01.2.2-LMT-K-718, Grant No. 01.2.2-LMTK-718-01-0014.

\section{References}

[1] G. Cerullo and S. De Silvestri, Ultrafast optical parametric amplifiers, Rev. Sci. Instrum. 74(1), 1-18 (2003), https://doi.org/10.1063/1.1523642

[2] D.D. Lowenthal, CW periodically poled $\mathrm{LiNbO}_{3}$ optical parametric oscillator model with strong idler absorption, IEEE J. Quantum Electron. 34(8), 1356-1366 (1998).

[3] S.C. Lyons, G.L. Oppo, W.J. Firth, and J.R.M. Barr, Beam-quality studies of nanosecond singly resonant optical parametric oscillators, IEEE J. Quantum Electron. 26(5), 541-549 (2000).

[4] G. Rustad, G. Arisholm, and Ø. Farsund, Effect of idler absorption in pulsed optical parametric oscillators, Opt. Express 19(3), 2815-2830 (2011).

[5] J. Ma, J. Wang, P. Yuan, G. Xie, K. Xiong, Y. Tu, X. Tu, E. Shi, Y. Zheng, and L. Qiani, Quasiparametric amplification of chirped pulses based on a $\mathrm{Sm}^{3+}$-doped yttrium calcium oxyborate crystal, Optica 2(11), 1006-1009 (2015), https://doi. org/10.1364/OPTICA.2.001006

[6] G. Valiulis, A. Dubietis, and A. Piskarskas, Optical parametric amplification of chirped X pulses, Phys. Rev. A 77, 043824 (2008), https:// doi.org/10.1103/PhysRevA.77.043824

[7] G. Arisholm, General numerical methods for simulating second-order nonlinear interactions in birefringent media, J. Opt. Soc. Am. B 14(10), 2543-2549 (1997), https://doi.org/10.1364 OSAB.14.002543 


\title{
OPTINIO PARAMETRINIO STIPRINIMO JUOSTOS IŠPLITIMAS DE்L SUGERIAMOS ŠALUTINĖS BANGOS
}

\author{
G. Valiulis, A. Varanavičius \\ Vilniaus universiteto Lazeriniu tyrimu centras, Vilnius, Lietuva
}

\begin{abstract}
Santrauka
Parametrinio šviesos stiprintuvo formuojamų impulsų trukmę lemia stiprintuvo stiprinimo juostos plotis. Parametrinio stiprinimo juostą nusako medžiagos dispersija ir sąveikos geometrinès ypatybès. Dažniausiai stiprinamų bangų sugertis yra laikoma žalingu reiškiniu stiprinimo procesui. Paaiškejjo, kad esant žymiems tiesiniams tik šalutinès bangos nuostoliams galima gauti net efektyvesnị parametrinị stiprinimą. Siame darbe
\end{abstract}

nagrinëjama šalutinès bangos tiesinių nuostolių įtaka parametriniam stiprinimui. Stiprinimo juosta buvo vertinama analiziškai sprendžiant tribangès parametrinès sąveikos lygtis plokščių bangų artinyje, esant monochromatiniam kaupinimui, neįskaitant kaupinimo nuskurdinimo. Pateikiami ir parametrinị stiprinimą aprašančių lygčių sprendimo pavyzdžiai, iliustruojantys sugerties įtaką stiprinimo juostos pločiui. 